\title{
Impacts of Jumps and Stochastic Interest Rates on the Fair Costs of Guaranteed Minimum Death Benefit Contracts
}

\author{
François Quittard-Pinon ${ }^{\mathrm{a}, \mathrm{b}}$ and Rivo Randrianarivony ${ }^{\mathrm{a}, \mathrm{b}}$ \\ ${ }^{a}$ Université de Lyon, Lyon, F-69003, France; Université Lyon 1, ISFA, 50, avenue Tony Garnier, \\ F-69366, France. \\ ${ }^{\mathrm{b}}$ EM Lyon Business School, 23, avenue Guy de Collongue, F-69134, France.
}

The authors offer a new perspective to the field of guaranteed minimum death benefit contracts, especially for simple return premium and rising floor guarantees. A particular feature of these contracts is a guaranteed capital upon the insured's death. A complete methodology based on the generalized Fourier transform is proposed to investigate the impacts of jumps and stochastic interest rates. This paper thus extends Milevsky and Posner (2001). If jumps alone are considered, similar results are obtained, but, when stochastic interest rates are introduced, the fair costs of the guarantee feature are found to be substantially higher in this more general economy.

The Geneva Risk and Insurance Review (2011) 36, 51-73. doi:10.1057/grir.2010.5; published online 15 June 2010

Keywords: life insurance contracts; variable annuities; guaranteed minimum death benefits; stochastic interest rates; jump diffusion models; mortality models

\section{Introduction}

Life insurance contracts have an actuarial and a financial component. The first is related to the remaining lifetime of the insured, the second is often linked to financial markets. These contracts generally offer a capital protection and a participation in the performance of the market. Thus, they match investors' desire to get protection in bear markets and upside participation in bull markets. Under different names, such as variable annuities (VA) in the U.S.A., segregated funds in Canada, unit-linked in the U.K., or other products, many kinds of policies bearing these features are offered to investors (see, for example, Hardy (2003) or Milevsky (2006)). In the present paper the authors study a specific contract, an insurance policy with an optional rider called a Guaranteed Minimum Death Benefit (GMDB). For convenience, such insurance policies are referred to as GMDB contracts. They belong to the class of VA and represent a multibillion dollar market in the U.S.A. 
The GMDB guarantees a specific monetary amount upon the insured's death. The contract is associated with a subaccount and the guarantee can take various predetermined forms. The usual one is the maximum of the subaccount value and of the initial investment accrued at a guaranteed rate. This guarantee is only triggered by the insured's death and is returned to the beneficiary of the policy. In this paper, we consider two types of GMDB contracts: the return-of-premium guarantee, and the rising floor. In the first case, the initial investment is guaranteed, while in the second case, a positive fixed return is guaranteed.

The initial premium is payable immediately to the insurance company that can invest it in financial markets. The promised guarantee, only paid on death, is not free but is paid to the insured by deducting small amounts from her subaccount. In practice, these payments are made on a periodic basis. In this paper, the modelling is in continuous time and contractual payments are made instantaneously. These fees are endogenously determined and are related by construction to the GMDB. In this paper, they will be referred to as insurance fees or insurance risk charges.

Milevsky and Posner (2001) found these contracts overpriced. In this paper, two possible explanations of this overpricing are examined. First, the authors observe that market financial prices can jump, creating a jump risk that must be taken into account. Second, interest rate risk must be incorporated in the valuation of these GMDB contracts.

The main objective of this paper is to determine to what extent these risk factors have an impact on the GMDB contract price. Is it only the jump risk which matters, the interest rate risk, or the combination of these risks? To examine this question, a new approach is introduced, which generalizes Milevsky and Posner's (2001) seminal article. Their valuation was performed in a Black and Scholes economy: the subaccount value was assumed to follow a geometric Brownian motion, and the term structure of interest rates was assumed to be constant. This last assumption, which can be acceptable for short-term options, is not acceptable for medium- or longterm contracts such as life insurance products. Generally, GMDB contracts are investment vehicles with a long-term horizon and, as such, they are very sensitive to interest rate movements that are by nature uncertain. A stochastic modelling of the term structure is therefore appropriate. In this paper, we use a one-factor model of a Hull and White (1993) type for stochastic interest rates.

The Gaussian hypothesis for asset returns has been questioned for a long time. It is now widely accepted that many observed return distributions display asymmetry and fat tails (see Cont, 2001). From a modelling point of view, the introduction of jumps allows these facts to be taken into account. The many crashes and rallies in financial markets over the last years show obvious jumps 
in prices. The suggested model accordingly incorporates upwards as well as downwards jumps.

In life insurance, Hardy (2003) suggested using regime-switching models introduced by Hamilton (1989), in particular the regime-switching lognormal model. Ballotta (2005) was the first author to analyze the impacts of jumps in valuing participating life insurance contracts, using a jump diffusion process with Gaussian jumps, while Kassberger et al. (2008) made use of Meixner and NIG processes. Le Courtois and Quittard-Pinon (2008) used a Kou process and took into account early default in with-profits life insurance contracts. In this paper, we also consider a Kou process, a jump diffusion process whose jumps have a double exponential distribution (see Kou, 2002).

In this paper, the impact of market risk and actuarial risk is analyzed through the "fair value" of insurance risk charges and of the GMDB contract. The fair value is the International Accounting Standards Board market valued recommendation for financial reporting. It can be expressed as the expectation of discounted cash flows under a particular probability measure, the so-called risk-neutral probability. This corresponds to the main result of arbitrage pricing theory. To sum up, we keep the framework of Milevsky and Posner and extend it by considering stochastic interest rates and jumps. Indeed, because of the long maturity of the contracts under study, the effects of the stochastic nature of interest rates are expected to be significant. And because of the nature of the embedded option, which is essentially a put option on the subaccount value, we anticipate that jumps increase the value of the fair insurance risk charge, especially if the probability of occurrence of downward jumps increases. We also expect a synergistic effect of jumps and stochastic interest rates. To understand why, consider the fact that as soon as we introduce stochastic interest rates, we can directly use zero-coupon bonds as numéraires. In this case, the subaccount relative price incorporates the characteristics of the term structure of interest rates, regardless of whether the interest rates and subaccount are correlated. We can intuitively infer from this fact that the uncertainty of interest rates captured by the volatility structure of zerocoupon bonds has an effect on the subaccount value, which, at the same time, can be lowered by negative jumps. This combined effect raises the embedded put option price. In fact, these interactions are very complex and only a suitable model can make the role of each of the economic variables involved explicit. This is precisely the purpose of the present paper.

In their article, Milevsky and Posner (2001) found insurance risk charges for simple return-of-premium contracts between one and ten basis points, while they reported a median value observed in the market of 115 basis points. In the numerical analysis of our model and using a particular set of parameters for 
the same type of contract, we found a range from 0.89 to 14.06 basis points insurance risk charge depending on purchase age when jumps are introduced. However, insurance risk charges range from 5.29 to 26.51 basis points when stochastic interest rates are considered on top of jumps. We found insurance fees ranging from 22.99 to 89.80 basis points for rising-floor contracts in the same setting.

We also show that with stochastic interest rates and a high probability of downward jumps, the model can yield high fees. So our model is versatile enough to obtain a large spectrum of prices. In particular, it allows us to recover the market values quoted by Milevsky and Posner (2001). Our results highlight the situations in which the combined effect of stochastic interest rates and jumps has a significant impact on insurance fees.

The remainder of this paper is organized as follows. The next section will introduce the main notation and the general framework used in the sequel. The pricing model and applications are studied in "Suggested pricing model" section. Various risk factor impacts are discussed in "Empirical study" section. The final section concludes the paper.

\section{General framework and main notations}

In this section, the main definitions and notations are formally introduced. The general framework of the analysis is set up. In "Financial risk and mortality" subsection, mortality and financial risk are considered. In "Contract payoff" subsection, the GMDB under analysis is defined. Subsection "Main equations" deals with the main equations of this paper while the last subsection reviews Milevsky and Posner's (2001) solution.

\section{Financial risk and mortality}

Financial risk is related to market risk firstly because the policyholder's account is linked to a financial asset or an index, and secondly via stochastic interest rates. We denote by $r$ the stochastic process modelling the instantaneous risk-free rate. The discount factor is

$$
\delta_{t}=e^{-\int_{0}^{t} r_{s} d s} .
$$

The policyholder's account value is modelled by the stochastic process $S$. In this model, $\ell$ stands for the fees associated with the insurance risk charge.

As far as mortality is concerned, we use the traditional actuarial notation. 
The remaining lifetime of a policyholder aged $x$ is a random variable $T_{x}$. For an individual aged $x$, the probability of death before time $t \geqslant 0$ is $P\left(T_{x} \leqslant t\right)=1-\left({ }_{t} p_{x}\right)$. Letting $\lambda$ denote the force of mortality, we have

$$
P\left(T_{x} \leqslant t\right)=1-\exp \left(-\int_{0}^{t} \lambda(x+s) d s\right) .
$$

As usual, $F_{x}(t)$ and $f_{x}(t)$ are, respectively, the c.d.f. and the p.d.f. of the random variable $T_{x}$. To ease notation, we generally omit the $x$ from the remaining lifetime random variable and only write $T$ when no confusion is possible. We assume stochastic independence between mortality and financial risks.

\section{Contract payoff}

The insurer promises to pay on the policyholder's death the contractual amount $\max \left\{S_{0} e^{g T}, S_{T}\right\}$, where $g$ is a guaranteed rate, $S_{0}$ is the insured initial investment, and $S_{T}$ is the subaccount value at time of death $x+T$. We will generalize this payoff further by considering a contractual expiry date $x+\Theta$. This contract only provides a guarantee on death. Otherwise, if the insured is still alive after time $\Theta$ lapses, she receives the account value at that time. For the sake of simplicity, we keep the first formulation, and we have

$$
\max \left\{S_{0} e^{g T}, S_{T}\right\}=S_{T}+\left[S_{0} e^{g T}-S_{T}\right]^{+} .
$$

Written in this way, the contract is seen to consist of a long position on the insured account plus a long position on a put option written on her account. Two remarks are in order: first, the insured not only has the same amount of money as if she invested directly in the financial market (aside from the fees), but also has the possibility of getting more, due to the put option. Second, because $T$ is a random variable, her option is not a vanilla one but an option whose exercise date is itself random (the insured's death). The other difference with the option analogy lies in the fact that, in this case, there is no upfront payment. Similarly to Milevsky and Posner (2001), the option part in (3) is referred to as the GMDB Option Payoff. Informally, we can write

$$
\text { Death Payment }=\text { Account Value }+ \text { GMDB Option Payoff. }
$$

In this contract, the investor pays the guarantee by installments. The paid fees constitute the so-called insurance risk charges. We assume that they are continuously deducted from the policyholder's account at a contractual proportional rate $\ell$. More precisely, we consider that in the time interval 
56

$(t, t+d t)$, the life insurance company receives $\ell S_{t} d t$ as instantaneous earnings. We denote by $F$ the cumulative discounted fees.

The contract can also be designed in order to cap the guaranteed rate $g$. In the VA literature, this is known as capping the rising floor. Let $C$ denote the maximum amount set in the policy by the insurer, $C \geqslant S_{0}$. In that case, the payoff becomes $\left[\min \left[C, S_{0} e^{g T}\right]-S_{T}\right]^{+}$. As Milevsky and Posner (2001) noticed, this can be further simplified to

$$
\left\{\begin{array}{lll}
\left(S_{0} e^{g T}, S^{T}\right)^{+} & \text {if } & T \leqslant \ln \left[\frac{C / S_{0}}{g}\right] \\
\left(C-S_{T}\right)^{+} & \text {if } & T>\ln \left[\frac{C / S_{0}}{g}\right] .
\end{array}\right.
$$

\section{Main equations}

In this paper, we are essentially interested in the fair value of the insurance risk charge. To evaluate it, we consider that the fair price is the arbitrage-free price which, according to arbitrage theory, is given by the expectation of the discounted contract payoff under a martingale measure that is equivalent to the physical or historical one. In our paper, we do not have a complete market. However, we suppose that a risk-neutral measure has been chosen. For practical purposes, it can be obtained from the market, see for example Björk (2004). There exists huge literature on how to choose a risk-neutral measure. This is not the purpose of this paper but the interested reader can find additional information, for example, in Cont and Tankov (2004) and references therein.

Using standard arbitrage theory in continuous time, the GMDB option fair price is

$$
G(\ell)=E_{Q}\left[\delta_{T}\left(S_{0} e^{g T}-S_{T}\right)^{+}\right],
$$

and upon conditioning on the insured's future lifetime,

$$
G(\ell)=E_{Q}\left[E_{Q}\left[\delta_{T}\left(S_{0} e^{g T}-S_{T}\right)^{+} \mid T=t\right]\right] .
$$

If $F_{T}$ denotes the discounted value of all fees collected up to time $T$, the fair value of the insurance risk charge can be written as

$$
M E(\ell)=E_{Q}\left[F_{T}\right]
$$

which, after conditioning, also gives

$$
M E(\ell)=E_{Q}\left[E_{Q}\left[F_{T} \mid T=t\right]\right] .
$$


As the protection is only triggered by the policyholder's death, the endogenous equilibrium price of the fees is the solution in $\ell$, if any, of the following equation

$$
G(\ell)=M E(\ell) .
$$

This is the key equation of this paper. To solve it, we have to define the investor account dynamics, and make additional assumptions on the process $S$, and, of course, on mortality.

\section{Milevsky and Posner solution}

In their paper, Milevsky and Posner (2001) assumed that $\ell$ is a constant, that $S$ is a Geometric Brownian motion with a volatility $\sigma$, and that the mortality is either of an exponential type or of a Gompertz type. With obvious notations, the GMDB option price, which they called a Titanic Option, is then given by

$$
G(\ell)=\int_{0}^{\Theta} f_{x}(t) E_{Q}\left[\delta_{T}\left(S_{0} e^{g T}-S_{T}\right)^{+} \mid T=t\right] d t,
$$

where the inner conditional expectation is exactly the standard Black Scholes Merton put formula with a strike price of $K=S_{0} e^{g t}$.

Assuming an exponential future lifetime, they obtained the price in closed form. Although this modelling is not realistic, it has the advantage of yielding simple formulas and it provides a kind of benchmark. Milevsky and Posner (2001) also obtained a closed-form solution for the present value of fees. For a parametrized version of Gompertz lifetime, numerical methods were necessary. In all cases, the equilibrium value of $\ell$ can only be obtained by a root searching algorithm.

\section{Suggested pricing model}

To investigate the GMDB contract fair price, the following model is introduced. First, the model takes stochastic interest rates into account. A one-factor interest rate model with an exponential volatility structure is used. ${ }^{1}$ Second, the underlying asset process incorporates jumps.

\footnotetext{
${ }^{1}$ This is a standard one-factor interest rate Hull and White (1993) model with a deterministic zero-coupon bond volatility structure of exponential type. In this model, interest rates are Gaussian. Therefore, negative interest rates can theoretically occur. However, when the model is calibrated using empirical data, the probability of these events is very low and not economically significant.
} 
The zero-coupon bond is assumed to obey the following stochastic differential equation (SDE) in the risk-neutral universe:

$$
\frac{d P(t, T)}{P(t, T)}=r_{t} d t+\sigma_{P}(t, T) d W_{t},
$$

where $P(t, T)$ is the price at time $t$ of a zero-coupon bond maturing at time $T, r_{t}$ is the instantaneous risk-free rate, and $\sigma_{P}(t, T)$ describes the volatility structure, and $W$ is a standard Brownian motion.

In order to incorporate a dependency between the subaccount and the interest rates, we suggest introducing a correlation between the diffusive part of the subaccount process and the zero-coupon bond dynamics. The underlying account price process $S$ is supposed to behave according to the following SDE under the chosen equivalent pricing measure $Q$ :

$$
\frac{d S_{t}}{S_{t^{-}}}=\left(r_{t}-\ell\right) d t+\rho \sigma d W_{t}+\sigma \sqrt{1-\rho^{2}} d Z_{t}+(Y-1) d \tilde{N}_{t} .
$$

Again, $r_{t}$ is the instantaneous interest rate, $\ell$ represents the fixed proportional insurance risk charge, $\sigma$ is the asset's volatility, $\rho$ is the correlation between the asset and the interest rate, $W$ and $Z$ are two independent standard Brownian motions, and the last term represents the jumps. $\tilde{N}$ is a compensated Poisson process with intensity $\lambda$, while $Y$, a random variable independent from the former processes, represents the price change due to a jump. The jump size is defined by $J=\ln (Y)$.

Let us emphasize here that the non-drift part $M$, defined by $d M_{t}=\rho \sigma d W_{t}+\sigma \sqrt{1-\rho^{2}} d Z_{t}+(Y-1) d \tilde{N}_{t}$, is a martingale in the considered risk-neutral universe.

\section{Modelling stochastic interest rates and subaccount jumps}

Denoting by $N_{t}$ the Poisson process with intensity $\lambda$ and applying Itō's lemma, the dynamics of $S$ can be expressed as

$$
S_{t}=S_{0} e^{\int_{0}^{t} r_{s} d s-\left(\ell+\frac{1}{2} \sigma^{2}+\lambda k\right) t+\rho \sigma W_{t}+\sigma \sqrt{1-\rho^{2}} Z_{t}+\sum_{i=1}^{N_{t}} \ln \left((Y)_{i}\right),}
$$

where $\kappa=E(Y-1)$.

A lengthy calculation shows that the subaccount price process can be derived under the $T$-forward measure as

$$
S_{t}=\frac{S_{0}}{P(0, t)} e^{X_{t}}
$$


where $X$ is the process defined by

$$
\begin{aligned}
X_{t}= & -\left(\ell+\frac{1}{2} \sigma^{2}+\lambda \kappa\right) t+\int_{0}^{t}\left(\left(\sigma_{P}(s, T)\left(\rho \sigma-\sigma_{P}(s, t)\right)+\frac{1}{2} \sigma_{P}^{2}(s, t)\right) d s\right. \\
& +\int_{0}^{t}\left(\rho \sigma-\sigma_{P}(s, t)\right) d W_{s}^{T}+\sigma \sqrt{1-\rho^{2}} Z_{t}+\sum_{i=1}^{N_{t}} \ln \left((Y)_{i}\right) .
\end{aligned}
$$

We can rewrite Eq. (11) as follows:

$$
\begin{aligned}
& S_{t}=S_{0} \exp \left(-\left(\ell+\frac{1}{2} \sigma^{2}+\lambda \kappa\right) t+\sigma \sqrt{1-\rho^{2}} Z_{t}\right) \\
& \times \underbrace{\frac{1}{P(0, t)} \exp \left(\int_{0}^{t}\left[\sigma_{P}(s, T)\left[\rho \sigma-\sigma_{P}(s, t)\right]+\frac{1}{2} \sigma_{P}^{2}(s, t)\right] d s+\int_{0}^{t}\left[\rho \sigma-\sigma_{P}(s, t)\right] d W_{s}^{T}\right)}_{\text {Stochastic interest rates }} \\
& \times \underbrace{\prod_{i=1}^{N_{t}} Y_{i}}_{\text {Jumps }}
\end{aligned}
$$

The expression of $S_{t}$ can be decomposed into three parts in the $T$-forward neutral universe. The first part is related to the subaccount trend and Brownian volatility, the second part is associated with the stochastic interest rates, while the last part takes the jumps into account.

This equation explains how the combined effect of jumps and stochastic interest rates works. We see that the stochastic interest rates, through the volatility $\sigma_{P}$, act as a multiplier on the jump part. We can also notice that the combined effect is present even if the correlation coefficient $\rho$ is equal to zero.

Present value of fees

By definition, $F_{t}$ is such that

$$
d F_{t}=\delta_{t} \ell S_{t} d t
$$

Using a chain rule on $\delta_{t} S_{t}$, we get

$$
F_{T}=\int_{0}^{T} d F_{t}=S_{0}-\delta_{T} S_{T}+\int_{0}^{T} \delta_{t} S_{t}-d M_{t} .
$$

Without loss of generality, we will also assume from now on that $S_{0}=1$. 
As the last term of $F_{T}$ is an integral with respect to a martingale, whose expectation is zero, we recover Milevsky and Posner's (2001) previous result on the present value of fees:

$$
M E(\ell)=E_{Q}\left[F_{T}\right]=1-E_{Q}\left[\delta_{T} S_{T}\right]
$$

Using (1) and (10), we get

$$
M E(\ell)=1-E_{Q}\left[e^{-\ell T-\frac{1}{2} \sigma^{2} T+\rho \sigma W_{T}+\sigma \sqrt{1-\rho^{2}} Z_{T}+\sum_{i=1}^{N_{T}} \ln \left((Y)_{i}\right)-\lambda \kappa T}\right] .
$$

As all random variables are independent from each other and by the properties of lognormal and compound Poisson laws, this reduces to

$$
M E(\ell)=1-E_{Q}\left[E_{Q}\left[e^{-\ell T} \mid T=t\right]\right]
$$

conditioning on the future lifetime $\mathrm{T}$,

$$
M E(\ell)=1-\int_{0}^{\infty} e^{-\ell t} f_{x}(t) d t
$$

where $f_{x}$ is the p.d.f. of the random variable $T$. A very interesting fact is that only the mortality model plays a role in the computation of the expected present value of fees, as seen in (14).

Introducing the time to contract expiry date $\Theta$, we have

$$
\begin{aligned}
M E(\ell) & =1-E_{Q}\left[e^{-\ell \min (T, \Theta)}\right] \\
& =1-E_{Q}\left[e^{-\ell T} \mathbb{1}_{\{T<\Theta\}}+e^{-\ell \Theta} 1_{\{T \geqslant \Theta\}}\right] \\
M E(\ell) & =1-\int_{0}^{\Theta} e^{-\ell T} f_{x}(t) d t-\left(1-F_{x}(\Theta)\right) e^{-\ell \Theta} .
\end{aligned}
$$




\section{Mortality models}

Two mortality models are considered, namely the Gompertz model and the Makeham model. ${ }^{2}$ In the case of the Gompertz mortality model, the force of mortality at age $x$ follows

$$
\lambda(x)=\frac{1}{b} \exp \left(\frac{x-m}{b}\right)
$$

where $m>0$ is the modal value of the Gompertz distribution and $b>0$ is a dispersion parameter.

After some algebra, the integral part in (15) can be written as

$$
\int_{0}^{\Theta} e^{-\ell T} f_{x}(t) d t=e^{b \lambda(x)} e^{(x-m) \ell}\left(\Gamma(1-\ell b, b \lambda(x))-\Gamma\left(1-\ell b, b \lambda(x) e^{\frac{\Theta}{b}}\right)\right)
$$

where $\Gamma(a, x)=\int_{x}^{\infty} e^{-t} t^{a-1} d t$ is the upper incomplete gamma function and $a$ must be positive. This condition entails an upper limit on the possible value of the insurance risk charge $\ell: \ell<1 / b$.

The present value of fees $^{3}$ in the case of a Gompertz-type mortality model amounts to

$$
\begin{aligned}
M E(\ell)= & 1-e^{b \lambda(x)} e^{(x-m) \ell}\left[\Gamma(1-\ell b, b \lambda(x))-\Gamma\left(1-\ell b, b \lambda(x) e^{\frac{\Theta}{b}}\right)\right] \\
& -e^{b \lambda(x)\left(1-e^{\frac{\Theta}{b}}\right)} e^{-\ell \Theta} .
\end{aligned}
$$

\section{Valuation of the embedded GMDB option}

The valuation of this embedded GMDB option is performed in two steps:

First, conditioning on the policyholder's remaining lifetime, the option is valued in the context of a Kou process with stochastic interest rates, with the assumption that the financial asset in the investor subaccount is correlated with the interest rates. This is a non-trivial problem which, to the best of our knowledge, has not been solved before.

${ }^{2}$ In the Makeham mortality model, the force of mortality is $\lambda(x)=\mathrm{A}+B C^{x}$, where $B>0, C>1$ and $A \geqslant-B$. The presence of the constant $A$ prevents us from obtaining closed-form formulas. Hence, a numerical quadrature was used to compute the present value of all collected fees.

${ }^{3}$ It is to be noted that the above formula (16) corrects typos in Milevsky and Posner's (2001) original article. 
Specifically, let us recall the embedded GMDB option fair price as can be seen in (4):

$$
G(\ell)=E_{Q}\left[E_{Q}\left[\delta_{T}\left(S_{0} e^{g T}-S_{T}\right)^{+} \mid T=t\right]\right] .
$$

Using the zero-coupon bond of maturity $T$ as a new numéraire, the inner expectation $I_{T}$ can be rewritten as

$$
I_{T}=E_{Q}\left[\delta_{T}\left(S_{0} e^{g T}-S_{T}\right)^{+}\right]=P(0, T) E_{Q_{T}}\left[\left(K-S_{T}\right)^{+}\right] .
$$

The last term of the right-hand side of this equation shows how using the forward-neutral measure $Q_{T}$ neutralizes the stochastic discount factor $\delta_{T}$. This expectation can now be computed using an adaptation of the generalized Fourier Transform approach ${ }^{4}$ proposed by Boyarchenko and Levendorskiî (2002).

Once this step is performed, the second step is to price the embedded GMDB option by quadrature using the density of a chosen mortality distribution. We consider two distributions, namely a parametrized Makeham, and a parametrized Gompertz distribution. The solution, although not in closed form, is fast and accurate. In this model, we consider that the two main sources of uncertainty come from the subaccount investment and from interest rates. Formally, these two risks are linked in (8) and (9). These equations lead to (11) for the risky subaccount price. In this expression, the impact of interest rates intervenes only through the zero-coupon bond volatility and through the initial term structure of interest rates. This modelling is a very parsimonious way to simultaneously take the two main risks into account and allows tractable solutions that we exploit in our empirical study.

\section{Empirical study}

Throughout this analysis, all mortality models were fitted from actual data. We systematically make a distinction between simple return-of-premium ( $g=0$ per cent) and rising-floor ( $g=5$ per cent) contracts.

\section{Jumps impact}

In this subsection, we only study the impact of jumps in the underlying account dynamics. The interest rate structure remains flat. The contract expiry is set at the age of 75 years. The mortality model is the Gompertz mortality model.

\footnotetext{
${ }^{4}$ A detailed account of the suggested generalized Fourier approach used in the paper can be obtained from the authors upon request.
} 
Table 1 Gompertz distribution parameters

\begin{tabular}{lccrr}
\hline Age (years) & \multicolumn{2}{c}{ Female } & \multicolumn{2}{c}{ Male } \\
\cline { 2 - 3 } & $m$ & $b$ & $m$ & $b$ \\
\hline 30 & 88.8379 & 9.213 & 84.4409 & 9.888 \\
40 & 88.8599 & 9.160 & 84.4729 & 9.831 \\
50 & 88.8725 & 9.136 & 84.4535 & 9.922 \\
60 & 88.8261 & 9.211 & 84.2693 & 10.179 \\
65 & 88.8403 & 9.183 & 84.1811 & 10.282 \\
\hline
\end{tabular}

Source: Milevsky and Posner (2001).

The Gompertz parameters used in this subsection and the next one are those calibrated to the 1994 Group Annuity Mortality Basic table in Milevsky and Posner (2001). They are recalled in Table 1.

We examine in turn the no-jumps case and two-jump diffusion models. The volatility in the no-jumps case is set to 20 per cent. In the Merton case, given here for comparison purposes, the jump sizes are Gaussian i.i.d. with mean $\mu_{J}$ and standard deviation $\sigma_{J}$. Here, $\mu_{J}=0$ and $\sigma_{J}=0.25$.

In the Kou case, the jump sizes $J=\ln (Y)$ are i.i.d. and follow a double exponential law:

$$
f_{J}(y)=p \eta_{1} e^{-\eta_{1} y} 1_{y>0}+q \eta_{2} e^{\eta_{2} y} 1_{y \leqslant 0}
$$

with $p \geqslant 0, q \geqslant 0, p+q=1, \eta_{1}>0$ and $\eta_{2}>0$. The parameter $p$ is the probability of upward jumps, and $q$ is the probability of downward jumps. The parameter $\eta_{1}$ is the inverse mean size of upward jumps while $\eta_{2}$ is the inverse mean size of downward jumps.

The following parameters for the Kou case serve as reference parameters: $p=0.4, \eta_{1}=10$, and $\eta_{2}=5$.

The Poisson intensity is arbitrarily set to $\lambda=0.5$ in both jump diffusion cases. The diffusive part of both jump diffusion models is such that their overall quadratic variation is exactly the same as the quadratic variation of the no-jumps case.

In Figure 1, we plotted the risk-neutral probability density functions of market returns according to the Kou model and according to the usual Gaussian case. We considered market returns over a 1-year period (see 1a), a 5-year period (see 1b), a 10-year period (see 1c), and a 15-year period (see 1d).

Tables 2 and 3 show the percentage of premium vs. the annual insurance risk charge in all three cases for a male policyholder (see Table 2 for the simple return of premium case and Table 3 for the policy with a rising-floor guarantee of 5 per cent). 
64

a

Risk-neutral pdf's for a time to expiry of $\tau=1$ year

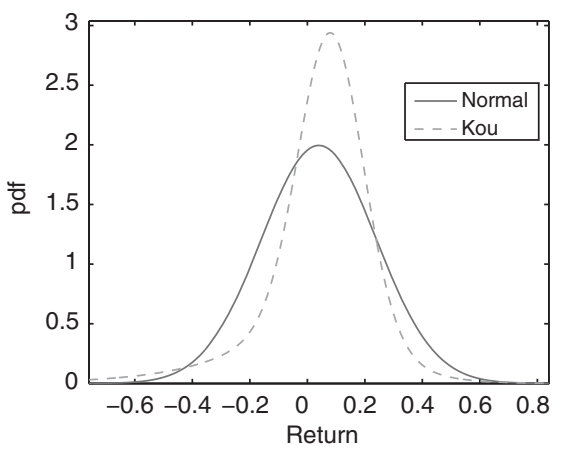

C

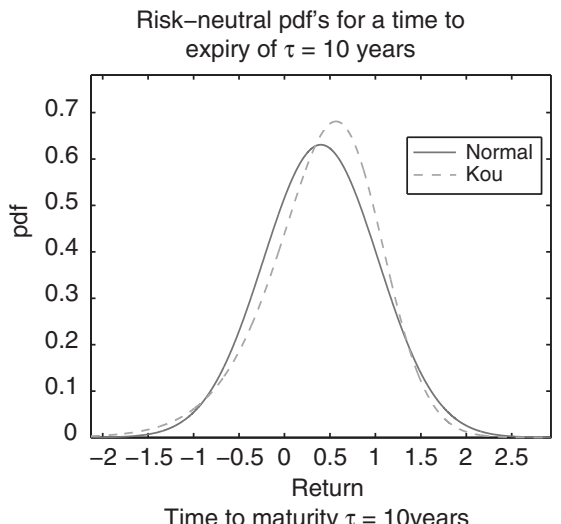

b

Risk-neutral pdf's for a time to expiry of $\tau=5$ years

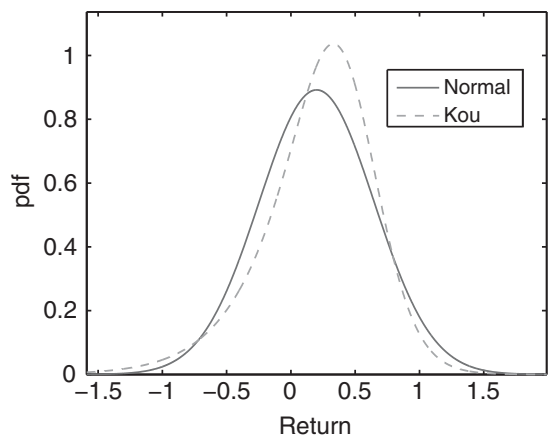

d

Time to maturity $\tau=5$ years

Risk-neutral pdf's for a time to expiry of $\tau=15$ years

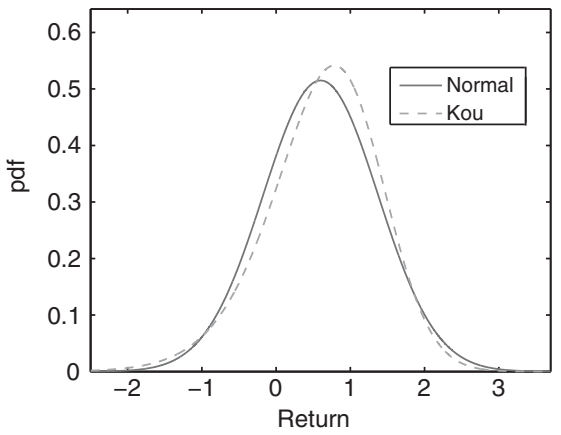

Time to maturity $\tau=15$ years

Figure 1. Risk-neutral densities for the Kou model with jump intensity $\lambda=0.5$ and jump component parameters $p=0.4, \eta_{1}=10$, and $\eta_{2}=5$ versus the Gaussian model with $\sigma=0.20$. The diffusive parameter in the Kou model is chosen to equate the total quadratic variation in both models.

We can notice that both jump diffusion models roughly give the same insurance risk charge, having the same overall quadratic variation. In the sequel, we only make use of the Kou process as a jump diffusion model.

\section{Stochastic interest rate impact}

Only stochastic interest rates are considered in this subsection.

The initial yield curve $y(0, t)$ is supposed to obey the following parametric equation $y(0, t)=\alpha-\beta e^{-\gamma t}$ where $\alpha, \beta$ and $\gamma$ are positive numbers. For 
Table 2 Jumps impact - male policyholder $-r=6 \%, g=0 \%, 200 \%$ cap

\begin{tabular}{|c|c|c|c|c|c|c|}
\hline \multirow[t]{2}{*}{ Purchase age (years) } & \multicolumn{2}{|c|}{ No jumps case } & \multicolumn{2}{|c|}{ Merton model } & \multicolumn{2}{|c|}{ Kou model } \\
\hline & $(\%)$ & $(b p)$ & $(\%)$ & $(b p)$ & $(\%)$ & $(b p)$ \\
\hline 30 & 0.25 & 0.61 & 0.25 & 0.60 & 0.25 & 0.60 \\
\hline 40 & 0.47 & 1.47 & 0.46 & 1.45 & 0.46 & 1.45 \\
\hline 50 & 0.82 & 3.64 & 0.79 & 3.54 & 0.79 & 3.52 \\
\hline 60 & 1.18 & 8.90 & 1.12 & 8.45 & 1.12 & 8.39 \\
\hline 65 & 1.19 & 13.24 & 1.10 & 12.32 & 1.09 & 12.19 \\
\hline
\end{tabular}

Gompertz mortality model. In each case, the left column displays the relative importance of the overall collected fees given by the ratio $\operatorname{ME}(\ell) / S_{0}$. The right column displays the annual insurance risk charge $\ell$ in basis points (bp).

Table 3 Jumps impact - male policyholder $-r=6 \%, g=5 \%, 200 \%$ cap

\begin{tabular}{|c|c|c|c|c|c|c|}
\hline \multirow[t]{2}{*}{ Purchase age (years) } & \multicolumn{2}{|c|}{ No jumps case } & \multicolumn{2}{|c|}{ Merton model } & \multicolumn{2}{|c|}{ Kou model } \\
\hline & $(\%)$ & $(b p)$ & $(\%)$ & $(b p)$ & $(\%)$ & $(b p)$ \\
\hline 30 & 1.35 & 3.25 & 1.34 & 3.24 & 1.24 & 2.98 \\
\hline 40 & 2.52 & 7.97 & 2.50 & 7.92 & 2.30 & 7.28 \\
\hline 50 & 4.23 & 19.22 & 4.19 & 19.05 & 3.85 & 17.44 \\
\hline 60 & 4.90 & 37.59 & 4.82 & 37.00 & 4.42 & 33.86 \\
\hline 65 & 3.48 & 39.33 & 3.38 & 38.16 & 3.10 & 34.99 \\
\hline
\end{tabular}

Gompertz mortality model. In each case, the left column displays the relative importance of the overall collected fees given by the ratio $M E(\ell) / S_{0}$. The right column displays the annual insurance risk charge $\ell$ in basis points (bp).

comparison purposes, we also use a flat interest rate structure set at $r=0.06$. The yield is then assumed to converge towards $r$ for longer maturities. The initial yield curve equation is set as follows:

$$
y(0, t)=0.0595-0.0195 \exp (-0.2933 t) .
$$

As stated earlier, the interest rate volatility structure is assumed to be of exponential form. It can be written as follows:

$$
\sigma_{P}(s, T)=\frac{\sigma_{P}}{a}\left(1-e^{-a(T-s)}\right),
$$

where $a>0$. In the sequel, we take $\sigma_{P}=0.033333, a=1$ and the correlation between the zero-coupon bond and the underlying account will be set at $\rho=0.35$. 
Table 4 Gompertz (G) and Makeham (M) mortality model parameters for the U.S.A.

\begin{tabular}{lccr}
\hline & $A$ & $B$ & $C$ \\
\hline$G_{U S}$ & & $6.148 \times 10^{-5}$ & 1.09159 \\
$M_{U S}$ & $9.566 \times 10^{-4}$ & $5.162 \times 10^{-5}$ & 1.09369 \\
\hline
\end{tabular}

Source: Melnikov and Romaniuk (2006).

Table 5 Stochastic interest rate impact on the annual insurance risk charge (bp) - No jumps considered - Gompertz mortality model $-g=5 \%, 200 \%$ cap

\begin{tabular}{lccccr}
\hline Age (years) & 30 & 40 & 50 & 60 & 65 \\
\hline Flat term structure & 4.79 & 11.16 & 24.88 & 44.45 & 45.20 \\
Stochastic interest rates & 4.49 & 10.64 & 24.27 & 44.78 & 46.81 \\
\hline
\end{tabular}

Melnikov and Romaniuk's (2006) Gompertz and Makeham parameters, estimated from the Human mortality database 1959-1999 mortality data, are used in the sequel. As shown in Table 4, no further distinction was made between female and male policyholders.

With these parameters and a rising-floor guarantee ( $g=5$ per cent), Table 5 shows that introducing stochastic interest rates does not change the insurance risk charge rate significantly. Thus, when taken separately, neither jumps nor stochastic interest rates have a significant effect on the fair insurance fees.

Indeed, the longer the time to maturity, the more jumps tend to smooth out, hence the lesser impact. On the other hand, the stochastic nature of interest rates has a significant impact for the typical time horizon involved in this kind of insurance contract. We study the combined effect of jumps and stochastic interest rates in greater depth in the following subsection.

\section{Impact of combined risk factors}

In subsequent figures, the circles correspond to the no-jumps model with a constant interest rate. The crosses correspond to the introduction of Kou jumps while maintaining a flat term structure of interest rates. The squares add jumps and stochastic interest rates to the no-jumps case. These three curves are built with a Gompertz mortality model. The stars take jumps and stochastic interest rates into account but the mortality model was replaced with a Makeham mortality model.

Figure 2 displays the annual insurance risk charge with respect to the purchase age in the case of a simple return-of-premium contract. From the age of 30 years to around 67 years (respectively 72 years), with stochastic interest 


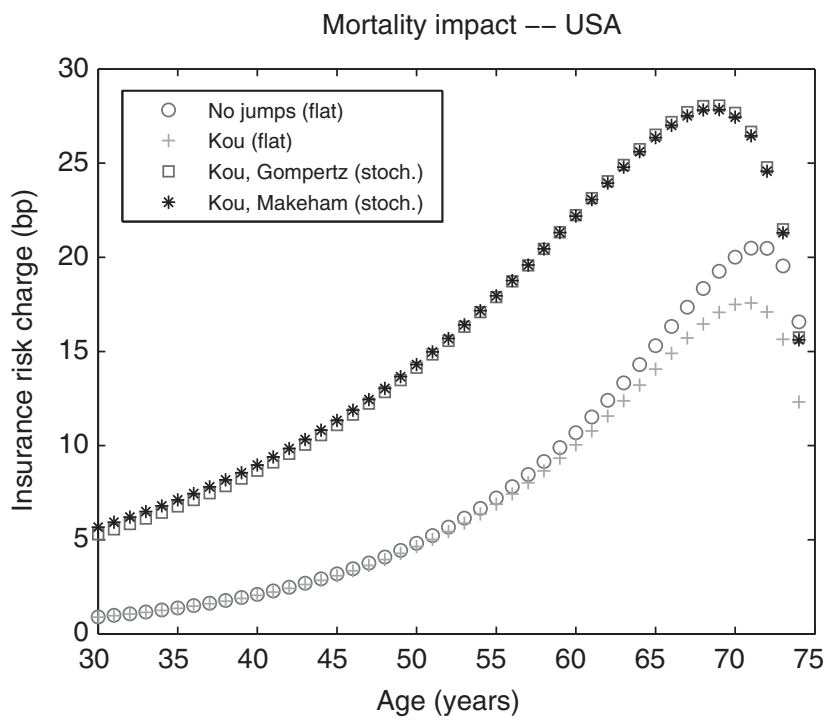

Figure 2. Annual insurance risk charge - U.S.A. $-g=0$ per cent.

rates (respectively with a flat term structure), the risk charge rises steadily across all models. It sharply decreases afterwards as the contract expiry approaches. All curves display a maximum.

Figure 3 presents the case with a rising-floor guarantee at 5 per cent. All curves exhibit the same pattern but the maximum is reached at younger ages, around 57 years in a stochastic interest rates environment and around 63 years in a flat term structure of interest rates environment.

More generally, the guarantee feature provided by the GMDB becomes less and less valuable as the purchase age nears the contract termination date. Indeed, the potential investor has no incentive to buy the GMDB policy if she is almost certain she won't possibly benefit from it in the short time left before contract expiry. Moreover, if the time horizon is short, the uncertainty surrounding the economic outlook is very low and she could make a profit by investing directly in a government bond.

The two lower curves in Figures 2 and 3 notably correspond to the flat term structure of interest rates setting. The jump effect is less pronounced than the stochastic interest rates effect as represented by the two upper curves. The thin band in which these upper curves lie shows that the change of mortality model also has much less impact than the stochastic nature of interest rates.

We also plotted the insurance risk charge for a GMDB contract with a simple return-of-premium guarantee as a function of the probability of upward 


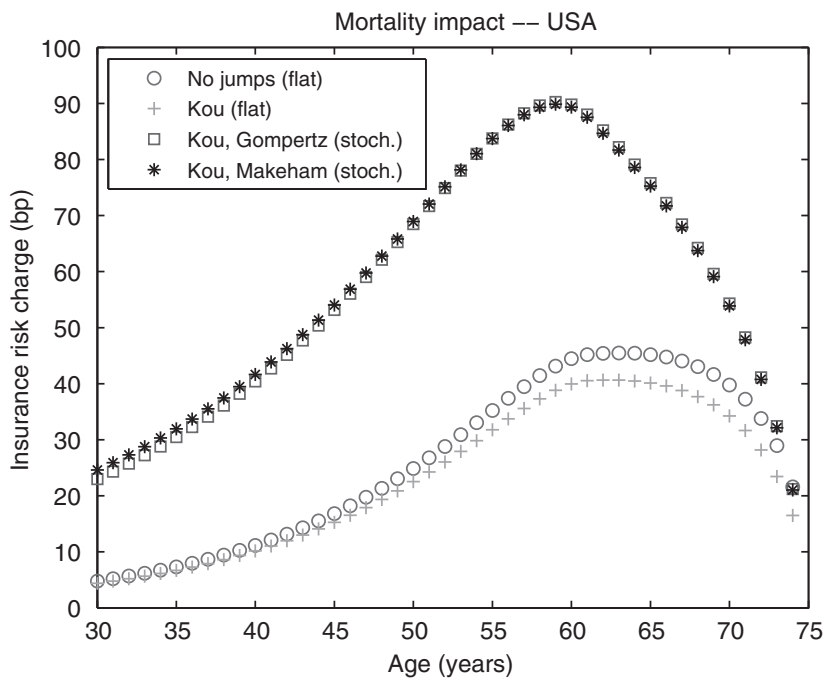

Figure 3. Annual insurance risk charge - U.S.A. $-g=5$ per cent.

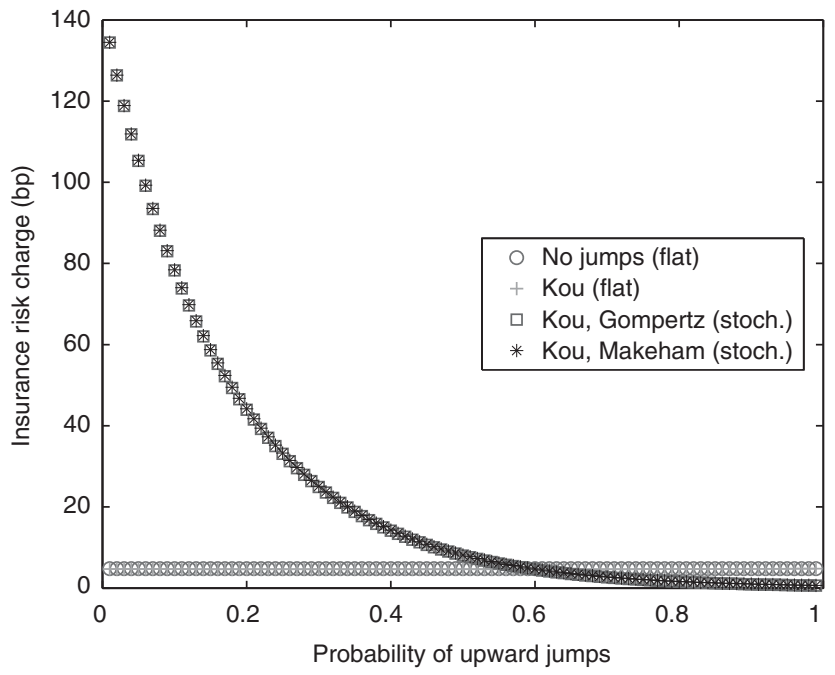

Figure 4. Sensitivity to the probability of occurrence of upward jumps.

jumps $p$ in Figure 4. The investor is 50 years old. Again, we can notice here that jumps alone don't change the insurance risk charge by much as the two curves in the flat term structures of interest rates setting can attest. Note that when we 
added stochastic interest rates alone to the Milevsky and Posner's (2001) model, the effect was not much more pronounced. However, once stochastic interest rates are introduced together with jumps, the impact of jumps is strongly reinforced, especially for lower probabilities of upward jumps $(p \leqslant 0.6)$. We can also see in Figure 4 that the fair insurance risk charge increases as the probability of occurrence of downward jumps increases. Moreover, the insurance fee can reach high levels (around $135 \mathrm{bp}$ ) for a very high probability of downward jumps. Thus, market fees reported by Milevsky and Posner (2001), with a median of $115 \mathrm{bp}$, can be recovered in our model. ${ }^{5}$ We can also observe that this combined effect is less important when upward jump probabilities increase. The fair insurance fees can even be lower than the ones predicted by the base model.

The various risk factor impacts are depicted in Figure 5 for a rising-floor guarantee ( $\mathrm{g}=5$ per cent) held by a policyholder aged 50 years. The full line in each subfigure corresponds to the present value of all collected fees $M E$ with respect to the insurance risk charge $\ell$. As noticed earlier, the present value of fees $M E(\ell)$ only depends on the remaining lifetime of the policyholder, which is modelled here by the Gompertz mortality model. The intersection of the fees line $(M E)$ with the embedded GMDB option value curves $(G)$ in each subfigure gives the fair value of the insurance risk charge $\ell$. Figure 5a represents the base case, with a flat term structure of interest rates and no jumps in the subaccount dynamics. The resulting fair charge is $24.88 \mathrm{bp}$. Figure $5 \mathrm{~b}$ shows the effect of adding only stochastic interest rates to this base case, whereas Figure $5 \mathrm{c}$ shows the impact of adding only jumps for various probabilities of upward jumps. The last Figure 5d shows the combined effect of both stochastic interest rates and jumps. A clear distinction can now be made between the three embedded GMDB option value curves $G(\ell)$. The fair insurance fee is at 39.89 bp for $q=50$ per cent, increases to $68.47 \mathrm{bp}$ for $q=60$ per cent, and finishes at $119.78 \mathrm{bp}$ for $q=70$ per cent.

This subsection is the most complete one because it takes into account jumps, stochastic interest rates and two standard mortality models estimated in the U.S.A. As reported in Tables 6 and 7, and displayed in Figures 2 and 3, the behaviour of the insurance risk charge with respect to age is of the same type whatever the considered model. However, within this type, differences can be noticed. First, the jump effect alone does not change the fees very much. Second, there are also substantial differences between the simple return-ofpremium and the rising-floor contracts. When the interest rate is constant and

\footnotetext{
${ }^{5}$ In light of the model developed in this paper, a possible explanation of these market fees would be that the insurers were adopting a very prudent attitude towards the financial markets, as if they expected high probabilities for downward jumps.
} 
a

GMDB option value vs. fees - No jumps - Gompertz (flat)

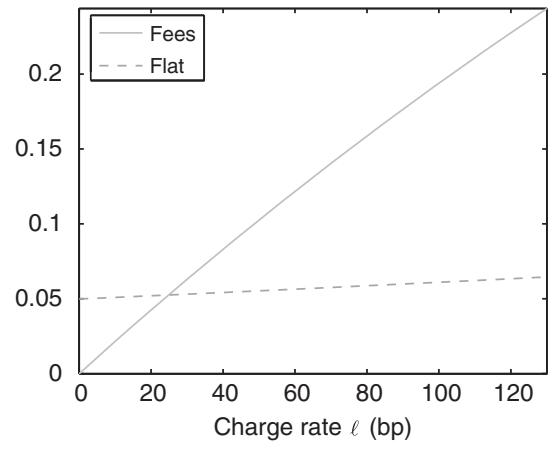

No jumps-Flat term structure

C GMDB option value vs. fees - Kou - Gompertz (flat)

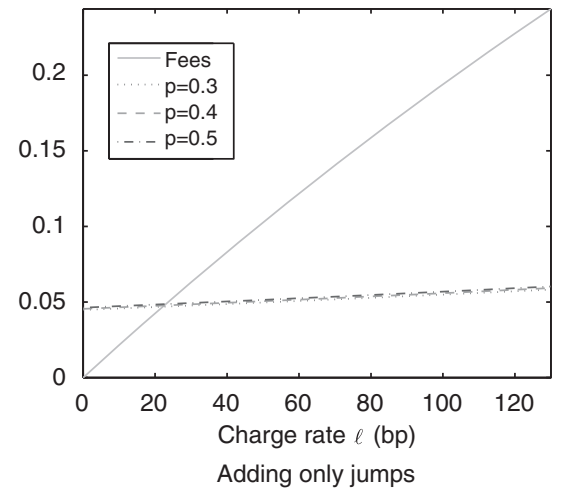

b GMDB option value vs. fees - No jumps - Gompertz (stoch)

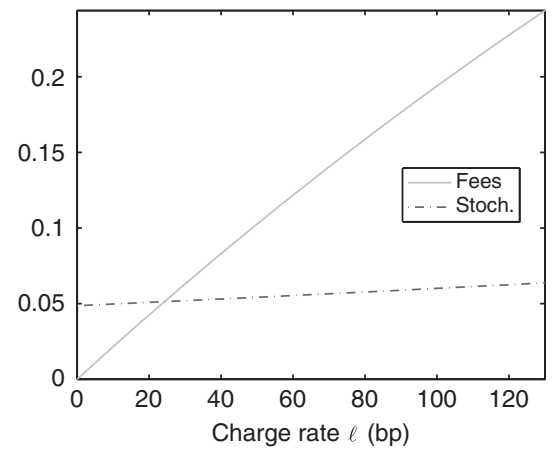

Adding only stochastic interest rates

d GMDB option value vs. fees - Kou - Gompertz (stoch.)

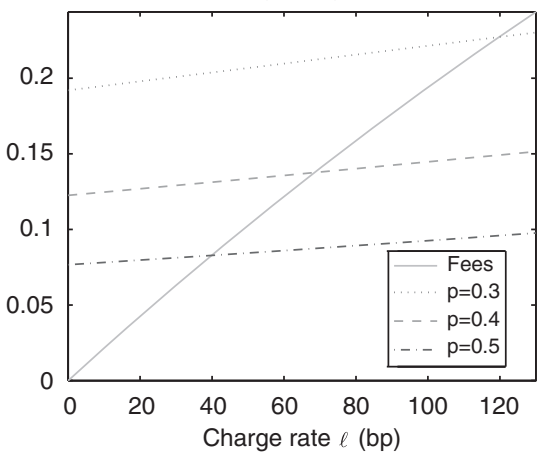

Combined effect of stochastic interest rates and jumps

Figure 5. Stochastic interest rate and jump impact on the fair value of both the total collected fees $M E$ and the embedded GMDB option $G$ with respect to the insurance risk charge $\ell$. The probability of upward jumps $p$ was set at $0.3,0.4$, and 0.5 .

a Kou process is considered, the results are close to those of Milevsky and Posner (2001) so jumps alone do not play a significant role. Third, when stochastic rates are combined with jumps, the results are very different: fees are substantially higher. Furthermore, and interestingly, the probability of upward jumps $p$ has a great influence: when $p$ decreases, fees rise as expected, as Figure 4 shows. On the one hand, for the simple return of premium contract, fees as high as $135 \mathrm{bp}$ are obtained for a 50 -year-old investor. On the other 
Table 6 Combined impact on the annual insurance risk charge (bp) - U.S.A. $-g=0 \%, 200 \%$ cap

\begin{tabular}{|c|c|c|c|c|c|}
\hline \multirow[t]{3}{*}{ Age (years) } & \multicolumn{4}{|c|}{ Gompertz } & \multirow{3}{*}{$\frac{\frac{\text { Makeham }}{\text { Kou }}}{\text { stoch. }}$} \\
\hline & \multicolumn{2}{|c|}{ No jumps } & \multicolumn{2}{|c|}{ Kou } & \\
\hline & flat & stoch. & flat & stoch. & \\
\hline 30 & 0.90 & 0.79 & 0.89 & 5.29 & 5.67 \\
\hline 40 & 2.10 & 1.90 & 2.05 & 8.67 & 8.97 \\
\hline 50 & 4.81 & 4.51 & 4.64 & 14.14 & 14.30 \\
\hline 60 & 10.68 & 10.51 & 10.04 & 22.24 & 22.19 \\
\hline 65 & 15.31 & 15.65 & 14.06 & 26.51 & 26.35 \\
\hline
\end{tabular}

Table 7 Combined impact on the annual insurance risk charge (bp) - U.S.A. $-g=5 \%, 200 \%$ cap

\begin{tabular}{|c|c|c|c|c|c|}
\hline \multirow[t]{3}{*}{ Age (years) } & \multicolumn{4}{|c|}{ Gompertz } & \multirow{3}{*}{$\frac{\frac{\text { Makeham }}{\text { Kou }}}{\text { stoch. }}$} \\
\hline & \multicolumn{2}{|c|}{ No jumps } & \multicolumn{2}{|c|}{ Kou } & \\
\hline & flat & stoch. & flat & stoch. & \\
\hline 30 & 4.79 & 4.49 & 4.39 & 22.99 & 24.61 \\
\hline 40 & 11.16 & 10.64 & 10.16 & 40.40 & 41.63 \\
\hline 50 & 24.88 & 24.27 & 22.52 & 68.47 & 68.92 \\
\hline 60 & 44.45 & 44.78 & 39.95 & 89.80 & 89.35 \\
\hline 65 & 45.20 & 46.81 & 40.13 & 75.78 & 75.25 \\
\hline
\end{tabular}

hand, fees lower than 1 bp can be obtained. Fourth, the choice of mortality model does not have a significant impact. ${ }^{6}$

\section{Conclusion}

In this paper, we consider the GMDB. In essence, upon death, this contract offers beneficiaries the maximum of the policyholder's initial capital accrued at a minimum guaranteed rate and the value of the insured account linked to a financial market. Milevsky and Posner (2001) named this contract a Titanic option. From a purely financial point of view, the pricing is done via contingent claim analysis. Previously, the fair contract price was obtained in a

\footnotetext{
${ }^{6}$ This observed fact corresponds to the drops of the mortality indices already mentioned by
} Melnikov and Romaniuk (2006). 
Black and Scholes context as in Milevsky and Posner (2001) or in a regimeswitching lognormal context as in Hardy (2003).

In this paper, the Black and Scholes framework is extended to a more general case allowing for stochastic interest rates and jumps. Specifically, the market value of the policyholder's account is assumed to follow a geometric Lévy process. We examine the case of jump diffusions. The mortality is of a Gompertz or a Makeham type. Our methodology was based on an adaptation of the generalized Fourier transform approach.

For the typical maturities involved in this kind of contract, we found that introducing jumps, while keeping the overall quadratic variation constant in a flat interest rate setting, doesn't change the fair costs of the GMDB very much and the results are similar to Milevsky and Posner's. On the other hand, introducing a stochastic interest rate setting raises these fair costs substantially. The important result stemming from this paper is that with stochastic interest rates significantly higher insurance risk charges are found, and this is even more pronounced for rising-floor contracts. The combined effect of stochastic interest rates and jumps is related to the probability of downward jumps: the higher this probability, the higher the fees.

\section{Conflict of interest}

The authors declare no conflict of interest

\section{Acknowledgements}

The authors thank François Le Grand, Richard Ruble, the GRIR editor, and two anonymous GRIR referees for helpful comments.

\section{References}

Ballotta, L. (2005) 'A Lévy process-based framework for the fair valuation of participating life insurance contracts', Insurance: Mathematics and Economics 37(2): 173-196.

Björk, T. (2004) Arbitrage Theory in Continuous Time, 2nd edn. Oxford Finance Series, USA: Oxford University Press.

Boyarchenko, S. and Levendorskiǐ, S. (2002) Non-Gaussian Merton-Black-Scholes Theory, Vol. 9 of Advanced Series on Statistical Science and Applied Probability, London: World Scientific, pp. xxi +398 .

Cont, R. (2001) 'Empirical properties of asset returns: Stylized facts and statistical issues', Quantitative Finance 1: 223-236.

Cont, R. and Tankov, P. (2004) Financial Modelling with Jump Processes, 2nd edn. London: Chapman \& Hall/CRC Press.

Hamilton, J.D. (1989) 'A new approach to the economic analysis of non stationary time series and the business cycle', Econometrica 57(2): 357-384. 
Hardy, M. (2003) Investment Guarantees: Modeling and Risk Management for Equity-linked Life Insurance, Hoboken, NJ, USA: John Wiley \& Sons.

Hull, J. and White, A. (1993) 'One-factor interest-rate models and the valuation of interest-rate derivative securities', The Journal of Financial and Quantitative Analysis 28(2): 235-254.

Kassberger, S., Kiesel, R. and Liebmann, T. (2008) 'Fair valuation of insurance contracts under Lévy process specifications', Insurance: Mathematics and Economics 42(1): 419-433.

Kou, S.G. (2002) 'A jump-diffusion model for option pricing', Management Science 48: 1086-1101.

Le Courtois, O. and Quittard-Pinon, F. (2008) 'Fair valuation of participating life insurance contracts with jump risk', Geneva Risk and Insurance Review 33: 106-136.

Melnikov, A. and Romaniuk, Y. (2006) 'Evaluating the performance of Gompertz, Makeham and Lee-Carter mortality models for risk-management with unit-linked contracts', Insurance: Mathematics and Economics 39: 310-329.

Milevsky, M.A. (2006) The Calculus of Retirement Income, Cambridge: Cambridge University Press.

Milevsky, M.A. and Posner, S.E. (2001) 'The titanic option: Valuation of the guaranteed minimum death benefit in variable annuities and mutual funds', The Journal of Risk and Insurance 68(1): $91-126$.

\section{About the Authors \\ François Quittard-Pinon, PhD and Docteur d'Etat, is a Professor of Finance at University Lyon 1, ISFA Actuary School, and EMLyon Business School.}

Rivo Randrianarivony, $\mathrm{PhD}$, is a research engineer at EMLyon Business School. 